4. Generalizations. The results for the wave equation can easily be generalized to include the inhomogeneous wave equation

$$
c^{2} \nabla^{2} u=\frac{\partial^{2} u}{\partial t^{2}}+F
$$

together with the mixed boundary-conditions

$$
u=U \quad \text { on } B_{1} \times(0, \infty), \quad \frac{\partial u}{\partial n}=V \quad \text { on } B_{2} \times(0, \infty)
$$

and the initial conditions (2.2). Indeed, all one has to do is add the terms

$$
\left(2 / c^{2} \int_{R} g * F * u(x, t) d x-2 \int_{B,} g * V * u(x, t) d x\right.
$$

to the right hand side of (2.10) and require that $K$ be the set of all functions $u$ which satisfy $u=U$ on $B_{1} \times(0, \infty)$. The analogous assertion applies to the heat conduction problem.

\title{
ReFerences
}

1. K. Washizu, Variational principles in continuum mechanics, Rept. No. 62-2, Dept. Aero. Engrg., University of Washington, 1962

2. M. E. Gurtin, Variational principles for linear elastodynamics, Archive Ratl. Mech. Anal. 16, 1, 1964

3. J. Mikusinski, Operational calculus, Pergamon Press, New York, 1959

\section{WAVE OPERATORS AND ABSOLUTELY CONTINUOUS SPECTRA*}

By C. R. PUTNAM (Purdue University)

1. On a Hilbert space $\mathfrak{S}$ of elements $f, g, \cdots$, with inner product $(f, g)$, let $\{E(\lambda)\}$, $-\infty<\lambda<\infty$, denote a one-dimensional spectral family. If $\mathfrak{S}_{a}$ denotes the Hilbert space spanned by the set of elements $f$ for which $\|E(\lambda) f\|^{2}$ is a absolutely continuous function of $\lambda$, then $\mathfrak{S}_{a}$ reduces the family $\{E(\lambda)\}$ and will be called the absolutely continuous part of $\mathfrak{S}$ determined by the spectral family $\{E(\lambda)\}$; see Halmos [1], p. 104, Kato [5], p. 240, Kuroda [6], p. 436. In the case of a self-adjoint operator $H$ or a unitary operator $U$, there exists in each instance a spectral family $\{E(\lambda)\}$ for which

$$
H=\int_{-\infty}^{\infty} \lambda d E(\lambda) \text { or } U=\int_{0}^{2 \pi} e^{i \lambda} d E(\lambda) .
$$

The restriction of $H$ (or $U$ ) to the corresponding space $\mathfrak{S}_{a}$ will be called the absolutely continuous part of $H$ (or $U$ ). The operator $H$ or $U$ will be called absolutely continuous on a subspace $\mathfrak{M}$ of $\mathfrak{S}$ if $\mathfrak{M} \subset \mathfrak{S}_{a}$.

If $\mathfrak{M}$ is a subspace its orthogonal complement in $\mathfrak{S}$ will be denoted by $\mathfrak{M}^{\perp}$. The space $\mathfrak{S}_{\mathfrak{l}_{1}}=\mathfrak{S}_{a}^{\perp}$ is spanned by the singular elements. Thus, if $f$ is in $\mathfrak{S}_{s}$ and if $f \neq 0$, then the absolutely continuous part of the monotone function $\|E(\lambda) f\|^{2}$ in the Lebesgue

${ }^{*}$ Received December 2, 1963. This work was supported by the National Science Foundation research grant NSF-GP1665. 
decomposition is absent, that is, the total variation of $\|E(\lambda) f\|^{2}$ occurs on a set of Lebesgue measure zero.

If $\mathfrak{M}$ is a linear manifold of $\mathfrak{S}$ its closure will be denoted by [M] . If $A$ is an operator its domain will be denoted by $\mathfrak{D}_{A}$ and its range by $\Re_{A}$.

Let $H_{0}$ and $V$ denote self-adjoint operators and suppose that

$$
H_{1}=H_{0}+V
$$

is also self-adjoint. For instance, if $V$ is bounded, its domain is $\mathfrak{S}$, and so $H_{1}$ is surely self-adjoint with domain identical to that of $H_{0}$. In the terminology of scattering theory, $H_{0}$ and $H_{1}$ correspond respectively to the free and total Hamiltonians, while $V$ is the interaction potential; see Jauch [2], p. 134, Jauch and Zinnes [3], p. 555.

2. It will henceforth be supposed that $H_{0}$ and $H_{1}$ are unitarily equivalent, so that there exists some unitary operator $U$ for which

$$
H_{1}=U H_{0} U^{*}, \text { or equivalently, } E_{1}(\lambda)=U E_{0}(\lambda) U^{*},
$$

where

$$
H_{0}=\int_{-\infty}^{\infty} \lambda d E_{0}(\lambda) \text { and } H_{1}=\int_{-\infty}^{\infty} \lambda d E_{1}(\lambda) .
$$

It is known that under certain hypotheses on $H_{0}$ and $V$ the strong limits

$$
U_{+}=\lim _{t \rightarrow \infty} U_{t} \text { and } U_{-}=\lim _{t \rightarrow-\infty} U_{t} \text {, where } U_{t}=\exp \left(i t H_{1}\right) \exp \left(-i t H_{0}\right),
$$

exist, are unitary, and satisfy

$$
H_{1}=U_{+} U_{0} U_{+}^{*} \text { and } H_{1}=U_{-} H_{0} U_{-}^{*} ;
$$

see [7] for references to Friedrichs, Kato, Kuroda, Rosenblum, et al.

There will be proved the following theorem, which is a generalization of a result in [8]:

(I) Let $H_{0}$ and $V$ be non-negative self-adjoint operators and suppose in addition that $V$ is bounded, thus

$$
H_{0} \geq 0, \quad 0 \leq V \leq k I(k=\text { const. }>0) .
$$

Suppose that $H_{1}$ is defined by (2) and that (3) holds for some unitary operator $U$. Let $\Gamma$ denote the smallest subspace of $\mathfrak{S}$ reducing $U$ and containing $\Re_{V}$. Then $U$ is absolutely continuous on $\Gamma$, that is,

$$
\Gamma \subset \mathfrak{S}_{a}(U) \text {. }
$$

In case $V$ is a perturbation of rank 1, it can be supposed that

$$
0 \leq V \leq k I \text {. }
$$

It follows from (I) that if $H_{0}$ is half-bounded, if $V$ is a one-dimensional perturbation, and if $H_{0}+V=U H_{0} U^{*}$ holds for some unitary $U$, then $U$ must have an absolutely continuous part. The corresponding assertion is clearly false for finite dimensional perturbations of rank different from 1, as can be seen by examples with finite matrices. Moreover, in this case, a simple trace argument shows that (3) and (9) cannot hold unless $k=0$. 
See Kato [5] who considers finite dimensional and, in some detail, one-dimensional perturbations of arbitrary (not necessarily half-bounded) self-adjoint operators.

3. Let $E_{n}$ denote Euclidean $n$-space and let $H_{0}$ denote the unique self-adjoint extension of the symmetric operator $-\Delta$ on $L^{2}\left(E_{n}\right)$, where $\Delta u \equiv \sum_{k=1}^{n} \partial^{2} u / \partial x_{k}^{2}$; see Kuroda [6], pp. 443-444 and Kato [4]. Then $H_{0}$ has the spectrum [0, $)$, hence $H_{0} \geq 0$, and, in addition, $H_{0}$ is absolutely continuous, that is (cf. (4)), $\mathfrak{S}_{a}\left(H_{0}\right)=\mathfrak{S}$. In case $n=1$ so that $V=V(x)$ is a one-dimensional multiplication operator satisfying $0 \leq V(x) \leq k(k=$ const. $>0)$, then (7) holds and $H_{1}$ of (2) is self-adjoint with a spectrum contained in $[0, \infty)$. Under certain restrictions on $V(x), H_{1}$ is also absolutely continuous with the spectrum $[0, \infty)$ and $(6)$ holds where $U_{+}$and $U_{-}$are unitary operators satisfying (5).

For instance, if $n=1$, so that

$$
H_{0}=-d^{2} / d x^{2} \text { on } \mathfrak{S}=L^{2}(-\infty, \infty),
$$

this situation holds if $V(x)$ satisfies

$$
0 \leq V(x) \leq k \text { and } \int_{-\infty}^{\infty} V(x) d x<\infty .
$$

Furthermore, in this case, if $V(x)$ satisfies the second relation of (11) and also

$$
0<V(x) \leq k
$$

almost everywhere, then the closure of $\Re_{V}$ is the entire space $\mathfrak{S}$. For this case, considered in $[8], \Gamma=\mathfrak{S}$, and so

$$
\mathfrak{S}_{a}(U)=\mathfrak{S},
$$

for each of the (unitary) wave operators $U=U_{+}$and $U=U_{-}$.

It was shown in [7] that the additional hypothesis

$$
\lim \inf b^{-3} \int_{b_{0}}^{b} V^{-1}(x) d x=0 \text { as } b \rightarrow \infty\left(b_{0} \text { fixed }\right)
$$

even assures that, for any unitary $U$ for which (3) holds, in particular for the wave operators,

$$
\text { the spectrum of } U \text { is the entire circle }|z|=1 \text {. }
$$

It can be remarked that even for arbitrary $n$, if $V$ is a radial potential, so that $V=V(r)$ with $r=\left(x_{1}^{2}+\cdots+x_{n}^{2}\right)^{1 / 2}$, which satisfies (12) and (14) with $x=r$, then, whenever (3) holds for some unitary $U$ (for instance, but not necessarily, by virtue of (6) in case $U_{+}$and $U_{-}$exist and are unitary), necessarily (13) and (15) hold. The sufficiency of (14) for (15) in case $V=V(r)$ can be proved by methods similar to those used for the case $n=1$.

4. In general, the relation (8) cannot be improved to

$$
\Gamma=\mathfrak{S}_{a}(U) \text {. }
$$

In fact, if $V=0$ then $\Gamma=0$ and (3) holds for any unitary $U$ commuting with $H_{0}\left(=H_{1}\right)$. In particular, such a unitary operator may be absolutely continuous. In the trivial case with $H_{0}=I\left(=H_{1}\right)$, relation (3) even holds for every unitary $U$. Another example is furnished by the absolutely continuous operator $H_{0}$ of $(10)$; in this case $U=\exp \left(i H_{0}\right)$ 
is absolutely continuous and commutes with $H_{0}$. On the other hand, for the case $V=0$ considered above, each of the wave operators $U=U_{+}$and $U=U_{-}$exists and is the identity $I$. Hence, in this case, $\left(8^{\prime}\right)$ does hold for $U=U_{+}$and $U_{-}$, since $\Gamma=\mathfrak{F}_{a}(I)$ is the space consisting of the element 0 only.

If $\Omega$ denotes the smallest subspace reducing $H_{0}$ and containing $\Re_{V}$, then also $\Omega$ reduces $H_{1}$ (see Kato [5]), hence also the wave operators, and so $\Gamma \subset \Omega$. Moreover, it is clear that the restriction of $U_{+}$and $U_{-}$to $\Omega^{\perp}$ is the identity operator. As a consequence of (I) there follows the theorem

(II) If, in addition to the hypotheses of $(I)$, the wave operators $U_{+}$and $U_{-}$of (5) exist and are unitary, then $U=U_{+}$or $U=U_{-}$has the direct sum representation

$$
U=U_{1} \oplus U_{2} \oplus I \quad \text { on } \quad \mathfrak{S}=\Gamma \oplus(\Omega \ominus \Gamma) \oplus \Omega^{\perp}\left(U=U_{+} \text {or } U_{-}\right),
$$

where $U_{1}$ is absolutely continuous. In the special case when $\Gamma=\Omega$, relation (16) can be refined to

$$
U=U_{a} \oplus I \quad \text { on } \quad \mathfrak{S}=\Omega \oplus \Omega^{\perp} \quad\left(U=U_{+} \quad \text { or } \quad U_{-}\right),
$$

where $U_{a}$ denotes the absolutely continuous part of $U$.

It will remain undecided whether either or both relations $\left(8^{\prime}\right)$ and

$$
\Gamma=\Omega
$$

must always hold if $U=U_{+}$or $U_{-}$. In the examples given earlier, with $H_{0}$ defined by (10) and $U=U_{+}$or $U=U_{-}$, then $\Gamma=\Omega=0$ when $V(x) \equiv 0$ and $\Gamma=\Omega=\mathfrak{S}$ when $V(x)>0$. By considering direct sums of Hilbert spaces, it is easy to construct examples. for which both $\left(8^{\prime}\right)$ and (17) hold for the wave operators, but where $\Gamma$ is a proper subspace of $\mathfrak{S}$.

5. Proof of (I). The proof will depend upon a modification of the argument given in [8]. Let $U$ have the spectral resolution

$$
U=\int_{0}^{2 \pi} e^{i \lambda} d E(\lambda)
$$

and let $F(\lambda)$ be a real-valued function of period $2 \pi$ with a continuous first derivative and possessing the Fourier series

$$
F(\lambda)=\sum_{-\infty}^{\infty} c_{k} e^{i k \lambda}=c_{0}+\sum_{1}^{\infty} c_{k} e^{i k \lambda}+\sum_{-1}^{-\infty} c_{k} e^{i k} .
$$

Since $F(\lambda)$ is real, $c_{-k}=\bar{c}_{k}$, and so

$$
F=c_{0}+F^{+}+\bar{F}^{+}, \text {where } F^{+}=\sum_{1}^{\infty} c_{k} e^{i k \lambda} .
$$

If $f$ is arbitrary and if $g$ is in $\mathfrak{D}_{H_{0}}$, then

$$
\left(\int_{0}^{2 \pi} F^{+}(\lambda) d E(\lambda) g, V^{1 / 2} f\right)=\left(\sum_{1}^{\infty} c_{k} V^{1 / 2} U^{k} g, f\right),
$$

and hence, if $h=V^{1 / 2} f$ and $\sigma(\lambda)=(E(\lambda) g, h)$ (cf. [8], middle of p. 845),

$$
\left|\int_{0}^{2 \pi} F^{+}(\lambda) d \sigma(\lambda)\right| \leq\|f\|\left(\sum_{1}^{\infty}\left|c_{k}\right|^{2}\right)^{1 / 2}\left(H_{0} g, g\right)^{1 / 2} \text {. }
$$


If the factors of the inner products of (21) are interchanged one obtains a relation corresponding to (22) but in which $\sigma(\lambda)$ is replaced by its conjugate. Since a complex number and its conjugate have the same absolute value, it follows that (22) remains valid if $F^{+}(\lambda)$ is replaced by $\bar{F}^{+}(\lambda)$. It then follows from (20) and (21) that

$$
\left|\int_{0}^{2 \pi} F(\lambda) d \sigma(\lambda)\right| \leq\left|c_{0}\right|\left\|V^{1 / 2} f\right\|\|g\|+2\|f\|\left(\sum_{1}^{\infty}\left|c_{k}\right|^{2}\right)^{1 / 2}\left(H_{0} g, g\right)^{1 / 2} .
$$

Hence,

$$
\left|\int_{0}^{2 \pi} F(\lambda) d(E(\lambda) g, h)\right|^{2} \leq C(f, g) \int_{0}^{2 \pi} F^{2}(\lambda) d \lambda,
$$

where $C(f, g)$ is a number depending on $f$ and $g$ but not on $F(\lambda)$. It follows that $(E(\lambda) g, h)$ is absolutely continuous whenever $g$ is in $\mathfrak{D}_{H_{\circ}}$ and $h$ is in $\Re_{V 1 / 2}$. Since $\mathfrak{N}_{H_{\mathrm{o}}}$ is dense in $\mathfrak{S}$, then $(E(\lambda) g, h)$ is absolutely continuous for all $g$ in $\mathfrak{S}$ and $h$ in $\Re_{V 1 / 2}$ and, in particular, $\|E(\lambda) h\|^{2}$ is absolutely continuous for all $h$ in $\Re_{V 1 / 2}$. Since $\left[\Re_{V 1 / 2}\right]=\left[\Re_{V}\right]$,

$$
\|E(\lambda) f\|^{2} \text { is absolutely continuous for all } f \text { in }\left[\Re_{V}\right] \text {. }
$$

If $g=U f$ then, by (18),

$$
\|E(\lambda) g\|^{2}=\int_{0}^{\lambda} d\|E(\mu) f\|^{2}, \quad 0 \leq \lambda \leq 2 \pi,
$$

and so $\|E(\lambda) g\|^{2}$ is absolutely continuous whenever $\|E(\lambda) f\|^{2}$ is. Similarly, the absolute continuity of $\|E(\lambda) f\|^{2}$ implies that of $\|E(\lambda) g\|^{2}$ whenever $g=U^{n} f(n=0, \pm 1, \pm 2, \cdots)$. Consequently, $\|E(\lambda) f\|^{2}$ is absolutely continuous for all $f$ in the closure of the linear manifold of finite linear combinations of elements $f_{n}$ in $\Re_{U n V}$. Since the set of such elements $f$ is clearly the space $\Gamma$, that is, the smallest subspace reducing $U$ and containing $\Re_{V}$, the proof of $(\mathrm{I})$ is complete.

\section{REFERENCES}

1. P. R. Halmos, Introduction to Hilbert space, Chelsea Pub. Co., New York (1951)

2. J. M. Jauch, Theory of the scattering operator, Helvetica Physica Acta 31 (1958) 127-158

3. J. M. Jauch and I. I. Zinnes, The asymptotic condition for simple scattering systems, I1 Nuovo Cimento 11 (1959) 553-567

4. T. Kato, Fundamental properties of Hamiltonian operators of Schrödinger type, Trans. Amer. Math. Soc. 70 (1951) 195-211

5. T. Kato, On finite dimensional perturbations of self-adjoint operators, Journal Math. Soc. Japan 9 (1957) 239-249

6. S. T. Kuroda, On the existence and unitary property of the scattering operator, I1 Nuovo Cimento 12 (1959) 431-454

7. C. R. Putnam, On the spectra of unitary half-scattering operators, Quarterly Appl. Math. 20 (1962) 85-88

8. C. R. Putnam, Absolute continuity of certain unitary and half-scattering operators, Proc. Amer. Math. Soc., 13 (1962) 844-846 